\title{
Relaciones entre el nivel de actividad física, experiencia deportiva e inteligencia emocional en alumnos de Grado de la Comunidad Madrid: Estudio de protocolo \\ Relation between physical activity levels, sport experience, and emotional intelligence among undergraduate students from Madrid Community: Protocol study \\ *,**Jorge Acebes-Sanchez **,***Gabriel Rodríguez-Romo \\ *Universidad Francisco de Vitoria (UFV); **Universidad Politécnica de Madrid (UPM), ***CIBERFES
}

\begin{abstract}
Resumen. A pesar de la variedad de enfoques ante el constructo de inteligencia emocional y la actividad física, se sigue investigando en esta área focalizando la atención en los deportistas actuales y en su rendimiento y no en las relaciones que estas actividades puedan tener con los diferentes niveles de las dimensiones de la inteligencia emocional. El objetivo principal de la investigación es estudiar las relaciones entre actividad física, experiencia deportiva a lo largo de la vida e inteligencia emocional en los alumnos universitarios. Se contactará con 1301 alumnos de Grado de la Comunidad de Madrid que contestarán a través de Google Forms a las siguientes herramientas: GPAQv2, encuesta ad hoc. De experiencia deportiva y TMMS-24. Se emplearán pruebas descriptivas, se llevará a cabo correlación bivariada de Pearson y se emplearán modelos de regresión múltiple por pasos. Los resultados ayudarán a enmarcar las investigaciones futuras dotando de importancia al deporte y a la actividad física como elementos fundamentales para el desarrollo y entrenamiento de forma transversal de las destrezas emocionales, encontrando y analizando las relaciones que existen entre el nivel de actividad física, la experiencia deportiva a lo largo de la vida de las personas y la inteligencia emocional en alumnos universitarios de Grado de la Comunidad de Madrid. Esta investigación pretende abrir una vía más en la que el deporte y la actividad física, vuelvan a ser el contexto idóneo para la solución de necesidades intrapersonales e interpersonales.
\end{abstract}

Palabras clave: Inteligencia Emocional, Actividad Física, Deporte, TMMS-24, GPAQ, Alumnos Universitarios.

\begin{abstract}
Despite the existence of a variety of approaches on emotional intelligence and physical activity, research currently focuses on sportsmen/sportswomen and their performance rather than on the relation between the latter and the different levels of emotional intelligence. The main objective of this research is to analyze the relation between physical activity, individuals long-life sports experience, and emotional intelligence among undergraduate students. We will contact 1,301 undergraduate students from the Community of Madrid, who will fill up different questionnaires on the platform Google Forms: GPAQv2, ad hoc survey of sports experience, and TMMS-24. We will show descriptive statistics, Pearson bivariate correlations, and stepwise multiple regression models. The results will help define future research, giving importance to sports and physical activity as main elements for the development and training of emotional skills, as well as finding and analyzing the relation between physical activity levels, long life sports experience, and emotional intelligence among undergraduate students of Community of Madrid. This research may open a new way for sports and physical activity to be the perfect context to satisfy intrapersonal and interpersonal needs.
\end{abstract}

Key Words: Emotional Intelligence, Physical Activity, Sport, TMMS-24, GPAQ, Undergraduate Students.

\section{Antecedentes}

Desde que Daniel Goleman popularizara el concepto de inteligencia emocional (1995), el cual fue acuñado por los psicólogos Salovey \& Mayer cinco años antes (1990), han sido numerosas las investigaciones que buscan relacionar la inteligencia emocional y el mundo de la actividad física y el deporte. Una revisión sobre el estado de investigación de la inteligencia emocional y el deporte encontró 60 artículos, tras los criterios de exclusión, publicados entre 2001 y 2012 (Ros, Moya-Faz, \& Garcés, 2013). Más tarde, una reciente revisión sistemática llevada a cabo por Laborde \& Doseville (2015) sobre los artículos empíricos que relacionaban la inteligencia emocional y la actividad física, el deporte o el ejercicio, encontrando 36 artículos objetivo tras los criterios de exclusión. De todos ellos, 30 eran sobre rendimiento deportivo y solo 6 sobre la relación con actividad física. Por último, es interesante destacar que se han llevado a cabo investigaciones relevantes en el contexto de la educación física (Fernández-Espínola \& Almagro, 2019; Fernández-García \& Fernández-Río, 2019). La mayoría de las publicaciones coinciden en el incipiente interés sobre el estudio de estas varia-

Fecha recepción: 02-12-18. Fecha de aceptación: 23-03-19

Jorge Acebes Sánchez

j.acebes.prof@ufv.es bles, No obstante, es importante resaltar que se observa una clara discordancia entre conceptualización teórica (inteligencia emocional como rasgo o inteligencia emocional como capacidad) (Pérez-González, Petrides, \& Furnham, 2014), herramientas de medida o metodología y diseño de las investigaciones.

Aunque existan definiciones muy extendidas y aceptadas como la descrita por Salovey y Mayer (1997), donde se expone y define la inteligencia emocional como una habilidad, se ha venido trabajando con el concepto «inteligencia emocional» refiriéndose a aspectos contrarios (Mestre, Comunian, \& Comunian, 2014). Por ello, como apuntan Extremera y Fernández-Berrocal (2014), debido a las diferentes opiniones sobre la definición del concepto inteligencia emocional y sus diferentes divisiones en dimensiones, han sido muchas las herramientas diferentes para su medición, encontrando dos grandes bloques: 1) Inteligencia emocional capacidad (Mayer \& Geher, 1996; Sullivan, 1999; Mayer, Salovey, \& Caruso, 2002) 2) Inteligencia emocional rasgo (Bar-On, 1997; Schutte, et al., 2001; Wong \& Law, 2002; Petrides, Pérez, \& Furnham, 2003). Pese a la dificultad de unificar criterios, se han encontrado relaciones entre las emociones y el rendimiento deportivo en líneas generales (Hanin, 2007), con ejercicio (Saklofske, Austin, Rohr, \& Andrews, 2007) y con los niveles de actividad física actual (Shuk-Fong, Lu, \& Hsiu-Hua, 2009; Vaquero-Solís, Amado Alonso, 
Sánchez-Oliva, SánchezMiguel, \& Iglesias-Gallego, 2018)

Analizando el peso de la revisiones bibliográficas y los diferentes enfoques de inteligencia emocional utilizados (rasgo y habilidad), cabe destacar que se están focalizando los esfuerzos en investigación sobre deportistas del presente, así lo demuestran las numerosas investigaciones que quieren aportar su propia herramienta de medición de la inteligencia emocional en el contexto deportivo (Lane, et al., 2009; Shuk-Fong et al, 2009; Arruza, González, Palacios, Arribas, \& Telletxea, 2013; Laborde, Dosseville, Guillén, \& Chávez, 2014) o incluso validaciones de herramientas existentes (GarcíaColl, Graupera-Sanz, Ruiz-Pérez, \& Palomo-Nieto, 2013).

\section{Objetivos}

El objetivo principal será estudiar las relaciones que existen entre el nivel de actividad física, la experiencia deportiva a lo largo de la vida de las personas y la inteligencia emocional en alumnos universitarios de Grado de la Comunidad de Madrid.

\section{Metodología/Diseño}

La presente investigación es un estudio transversal. Los participantes pertenecientes al objeto de la muestra estratificada, colaborarán en el estudio de manera voluntaria contestando dos cuestionarios ampliamente validados y una encuesta ad hoc. validada por juicio de expertos. Todos ellos se cumplimentarán a través de una herramienta informática que ha sido expuesta ante una validación de expertos para

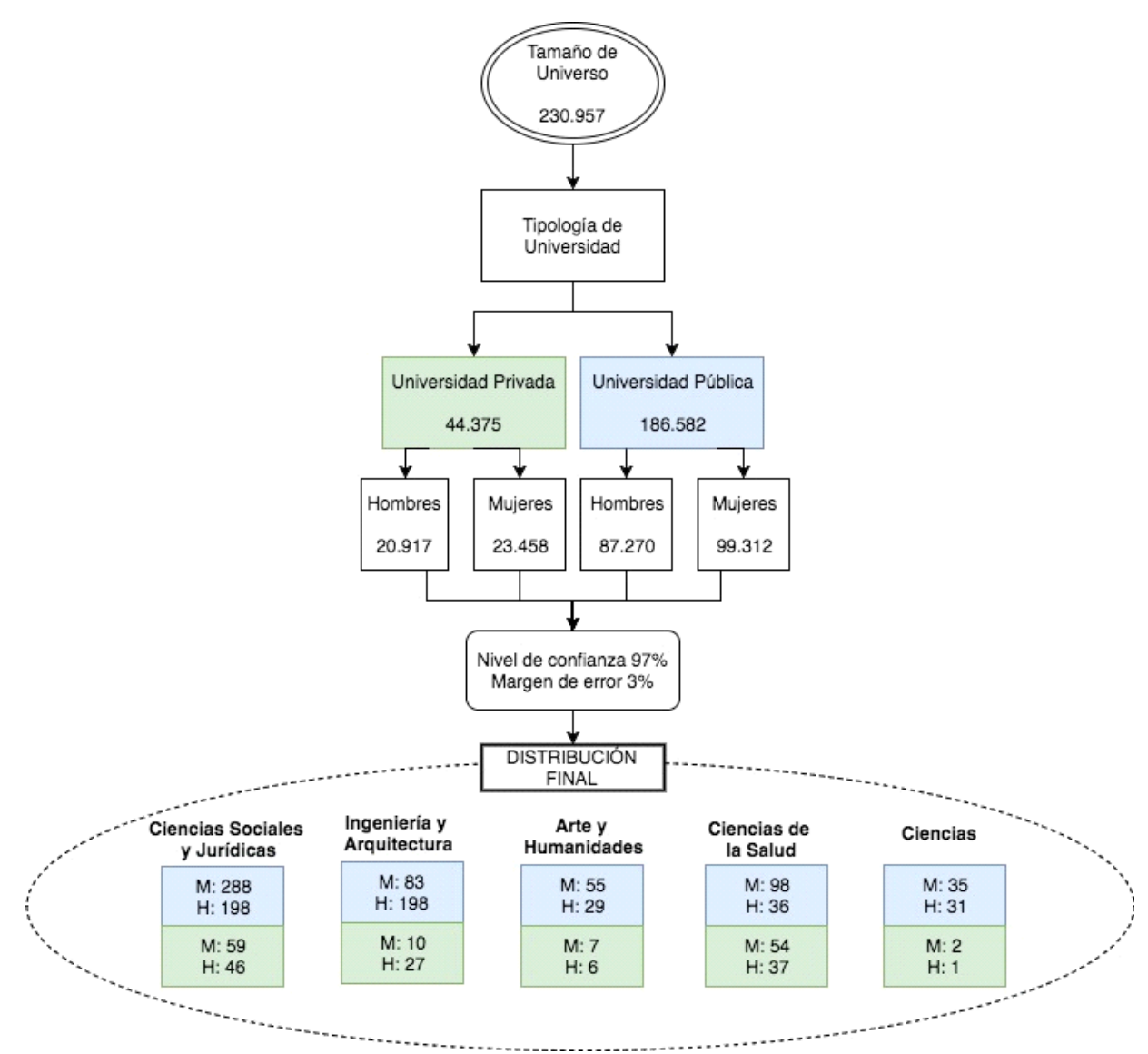

Figura 1. Distribución final de la muestra. verificar que no existen diferencias entre la cumplimentación de los cuestionarios en formato origen y formato digital.

\section{Participantes del estudio}

Todos los participantes del estudio son alumnos y alumnas de Grado de la Comunidad de Madrid. Siendo su participación voluntaria, se realizará un muestreo estratificado en base a las diferentes variables: género (hombre o mujer), áreas de conocimiento del Grado (Ciencias Sociales y jurídicas, Ingeniería y Arquitectura, Artes y Humanidades, Ciencias de la Salud y Ciencias) y tipología de Universidad (pública o privada)

\section{Tamaño de la muestra}

Teniendo en cuenta que el tamaño del universo de la muestra es de 230.957 sujetos, asumiendo un margen de error del 3\% y un nivel de confianza del 97\%, se considera significativo un muestreo estratificado de 1301 participantes (692 mujeres y 609 hombres). La distribución final de la muestra se refleja en la figura 1.

\section{Herramientas de medida}

Para el estudio se van a utilizar tres herramientas: TMMS24, GPAQv2 y la encuesta de experiencia deportiva.

\section{TMMS-24}

Para la evaluación de la inteligencia emocional se utilizará la versión española modificada del Trait Meta-Mood Scale, ya validada por Fernández-Berrocal, Extremera \& Ramos (2004) y que trata la inteligencia emocional como rasgo, se decide utilizar esta herramienta debido al tamaño muestral. Esta medida de autoinforme se compone de 24 ítems con una escala tipo Likert de 5 puntos. Al igual que la original, compuesta por tres dimensiones (ocho ítems cada una): Atención, claridad y reparación emocional, mostrando una consistencia interna de: atención (á=0,90), claridad (á $=0,90)$ y reparación emocional (á = 0,86). Estos componentes se definen del siguiente modo:

Atención: Soy capaz de sentir y expresar los sentimientos de forma adecuada.

Claridad: Comprendo bien mis estados emocionales.

Reparación: Soy capaz de regular los estados emocionales correctamente.

Para corregir y obtener una puntuación en cada uno de los factores, se sumarán los ítems del 1 al 8 para el factor atención emocional, los ítems del 9 al 16 para el factor claridad emocional y del 17 
al 24 para el factor reparación de las emociones. A continuación, se cruzarán los datos con las tablas de evaluación de la herramienta teniendo en cuenta que habrá diferencia de género a la hora de la corrección.

\section{GPAQv2}

Para la evaluación del nivel de actividad física se utilizará la versión 2 del Global Physical Activity Questionnaire (GPAQv2), cuestionario validado y constituido por 16 preguntas para medir el nivel de actividad física actual de los participantes (Cleland, Hunter, Kee, Cupples, Sallis, \& Tully, 2014). Este cuestionario ha sido utilizado para estudios epidemiológicos en torno a 50 países (Leal, et al., 2009).

El cuestionario aporta información sobre la intensidad (vigorosa o moderada), la frecuencia (días de práctica en una semana típica) y la duración (horas y minutos en un día típi- co) de la actividad física desarrollada en el trabajo, estudios, tareas domésticas o búsqueda de trabajo. Los desplazamientos (caminando o en bicicleta). Y la actividad desarrollada en el tiempo libre (ocio). Además, una pregunta profundizará en el comportamiento sedentario de los participantes.

\section{Encuesta de experiencia deportiva}

Para la recogida de información sobre la experiencia deportiva de los participantes, se ha desarrollado una encuesta de elaboración propia basado en la entrevista de la «Encuesta de hábitos deportivos en España de 2015».

Para la validación de la encuesta se ha contado con la colaboración de 15 expertos que han llegado a consenso tras 2 rondas de modificaciones.

Esta encuesta recoge la información de la experiencia deportiva de las personas a lo largo de la vida, de esta mane-

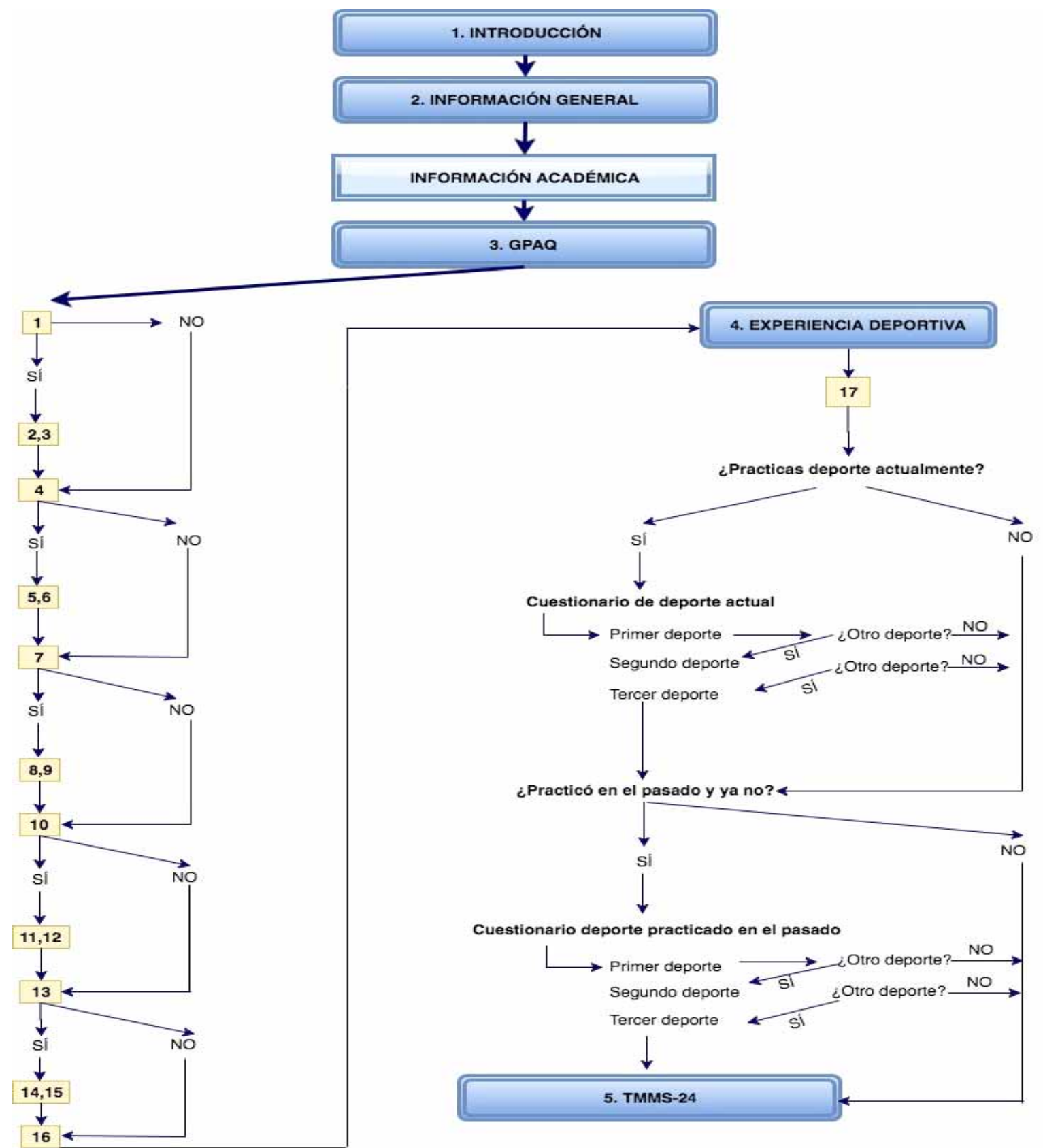

Figura 2. Diagrama de flujo. Cumplimentación del cuestionario. 
ra, no solo hace referencia a las actividades físico-deportivas practicadas en el momento actual, sino que también recoge información sobre aquellas actividades que se practicaron en el pasado.

La información recogida por esta encuesta es: número de deportes practicados, tipo de deportes, años de práctica, frecuencia semanal de práctica, nivel máximo de competición alcanzado y años de competición a dicho nivel, de cada una de las actividades físico-deportivas seleccionadas.

\section{Herramienta de recogida de datos}

Los participantes, de manera voluntaria, cumplimentarán el cuestionario compartido a través de Google Forms. A continuación, se muestra la secuencia del cuestionario (Figura 2):

1. Introducción.

Los participantes analizan si cumplen los requisitos para la elaboración del cuestionario, así como descubren la información genérica del mismo.

2. Información General.

Primera información a cumplimentar, donde se definen las características que estratifican la muestra: Universidad privada o pública, área de conocimiento y género. Además, se solicita información genérica sobre el perfil físico de los participantes (altura y peso) y rendimiento académico.

\section{GPAQv2.}

Cuestionario validado con 16 preguntas condicionadas que la propia herramienta gestiona de manera automática sobre el nivel de actividad física del participante en base a: nivel de actividad física en el trabajo/universidad, nivel de actividad física en los desplazamientos, nivel de actividad física en el tiempo libre y una pregunta sobre el comportamiento sedentario

4. Encuesta de experiencia deportiva.

Encuesta que se refiere a los deportes o actividades físico-deportivas más practicadas en el momento actual, contestadas por orden de importancia y refiriéndose a las preguntas hasta un máximo de 3 deportes o actividades físicodeportivas actuales, dando paso a los deportes o actividades físico-deportivas practicadas solo en el pasado.

\section{TMMS-24.}

Cuestionario con 24 ítems sobre inteligencia emocional que mide: atención, claridad y reparación emocional.

Manejo de los datos. Adquisición, gestión y transferencia de los datos

Todos los datos de los participantes se recogerán a través de la herramienta Google Forms. Los participantes del estudio se identificarán bajo una nomenclatura anónima, en ningún momento aportarán datos de carácter identificativo. Los datos recogidos se mantendrán bajo estricta confidencialidad con el único propósito de su utilización en la investigación en curso. A su vez, se mantendrán en lugar seguro al amparo de la legislación vigente para tal efecto.

Los datos obtenidos de los cuestionarios se filtrarán, tabularán y codificarán para su posterior análisis en Statistical Package for the Social Science (SPSS v21).

\section{Análisis estadístico}

El análisis estadístico busca esclarecer qué diferencias existen entre las variables, realizando comparaciones entre las mismas. En primer lugar, se realizará un análisis descriptivo para explorar las características de la muestra. Para ello se emplearán pruebas descriptivas ANOVA para variables numéricas y Chi Cuadrado para variables categóricas. Si los resultados de estas pruebas son significativos $(\mathrm{p}<.05)$ se llevará a cabo correlación bivariada de Pearson y se emplearán modelos de regresión múltiple jerárquicas por pasos analizando las siguientes variables: Nivel de actividad física, tipo de deporte practicado (individual, colectivo o de combate), años de práctica, frecuencia de prácticas semanal y nivel máximo de competición. Para determinar el tamaño de los efectos encontrados, se utilizarán $c_{\mathrm{p}}^{2} \mathrm{y} \mathrm{r}^{2}$, asumiendo valores inferiores a 0,06 como efecto pequeños, valores entre 0,06 y 0,14 como efecto moderados y tamaños superiores a 0,14 como efecto grande.

\section{Discusión}

Partiendo de la base de que uno de los mayores intereses sobre la inteligencia emocional ha estado tradicionalmente ligado al mundo empresarial y al rendimiento en el trabajo, encontrándose relaciones significativas entre ambas variables (Travis \& Lac, 2006), así como con el rendimiento académico (Buenrostro-Guerrero, Valadez-Sierra, Soltero-Avelar, Nava-Bustos, Zambrano-Guzmán, \& García-García, 2012), existe una necesidad en encontrar e identificar qué puede entrenar y mejorar los niveles de inteligencia emocional. Algunos autores manifiestan que percibir y expresar emociones, comprender las emociones o ayudar a las personas a tener estrategias de afrontamiento para su regulación emocional y plantear alternativas, son las estrategias idóneas para mejorar los niveles de inteligencia emocional y así repercutir en la mejora de rendimiento de estas personas (Lopes, Côté, \& Salovey, 2014). Es por lo tanto evidente, que las estrategias planteadas siempre han ido en la línea del trabajo de estas habilidades de forma analítica para analizar la repercusión en el rendimiento.

Las actividades físico-deportivas presentan el contexto idóneo para plantear la percepción y expresión de emociones, comprensión emocional o recrean situaciones de una necesaria regulación emocional. De cara a la aportación que hacen las actividades físico-deportivas a la inteligencia emocional, cabe destacar que se plantean diferentes resultados por tipología deportiva en relación a las diferentes dimensiones de la inteligencia emocional, así como la intensidad de la correlación se cree que guarda relación con el nivel competitivo. Se ha demostrado que el trabajo de coaching y mejora de las dimensiones de la inteligencia emocional, está relacionado con un mejor rendimiento deportivo (Barlow \& Banks, 2014; Crombie, 2011) Cabe destacar que el deporte no desarrollará la inteligencia emocional per se, si no que necesitará de la regulación de los profesionales involucrados para tal fin. Existen estudios en los que se expone que en algunas situaciones los resultados pueden ser negativos (Lopes, Barsade, Nezlek, Elfenbein, Straus, \& Salovey, 2003).

\section{Conclusión}

Se esperan encontrar relaciones significativas entre los diferentes deportes, niveles de actividad física y las diferentes dimensiones de la inteligencia emocional, así como un 
tamaño del efecto entre moderado y grande. Aunque se deben seguir explorando las relaciones entre la práctica deportiva, el nivel de rendimiento o las horas invertidas a lo largo del tiempo en deporte o actividad física y su relación con la inteligencia emocional, encontrar resultados positivos relacionales en este ámbito, validarían la hipótesis de que el deporte y la actividad física plantean los escenarios idóneos para el desarrollo y práctica de situaciones que estén relacionadas con el entrenamiento de la inteligencia emocional en sus diferentes dimensiones.

\section{Referencias}

Arruza, J., González, O., Palacios, M., Arribas, S., \& Telletxea, S. (2013). Un modelo de medida de la Inteligencia Emocional percibida en contextos deportivo/competitivos. Revista de Psicología del Deporte, 22(2), 405-413.

Bar-On, R. (1997). Emotional Quotient Inventory (EQ-i): A mesure of emotional intelligence. (T. M.-H. Systems, Ed.)

Barlow, A., \& Banks, A.P. (2014). Using emotional intelligence in coaching high-performance athletes: a randomised controlled trial. Coaching: An International Journal of Theory, Research and Practice, 7(2), 132-139.

Buenrostro-Guerrero, A., Valadez-Sierra, M., Soltero-Avelar, R., Nava-Bustos, G., Zambrano-Guzmán, R., \& García-García, A. (2012). Inteligencia emocional y rendimiento académico en adolescentes. Revista de Educación y Desarrollo, 20, 29-37.

Cleland, C., Hunter, R., Kee, F., Cupples, M., Sallis, J., \& Tully, M. (2014). Validity of the Global Physical Activity Questionnaire (GAPQ) in assessing levels and change in moderate-vigorous physical activity and sedentary behaviour. BMC Public Health, 14.

Consejo Superior de Deportes (2015). Encuesta de hábitos deportivos en España 2015. Ministerio de Educación, Cultura y Deporte , Subdirección General de Estadística y Estudios, Secretaría General Técnica.

Crombie, D. (2011). The role of emotional intelligence in sports performance. University of Cape Town, S.

Extremera, N., \& Fernández-Berrocal, P. (2014). Una guía práctica de los instrumentos actuales de evaluación de la inteligencia emocional. En J. Mestre Navas, \& P. Fernández-Berrocal, Manual de inteligencia emocional (págs. 99-122). Madrid: Ediciones Pirámide.

Fernández-Berrocal, P., Extremera, N., \& Ramos, N. (2004). Validity and reliability of the Spanish Modified Version of the Trait Meta-Mood Scale. Psychological Reports, 94, 751-755.

Fernández-Espínola, C., \& Almagro, B.J. (2019). Relación entre motivación e inteligencia emocional en Educación Física: una revisión sistemática. RETOS, 36.

Fernández-García, L., \& Fernández-Río, J. (2019). Proyecto Wonderwall: identificación y manejo de emociones en la Educación Física de Educación Primaria. Retos, 35.

García-Coll, V., Graupera-Sanz, J.-L., Ruiz-Pérez, L.-M., \& Palomo-Nieto, M. (2013). Inteligencia Emocional en el Deporte: Validación española del Schutte Self Report Inventory (SSRI) en deportistas españoles. Cuaderno de Psicología del Deporte, 13(1), 25-36.

Goleman, D. (1995). Emotional Intelligence. New York: Bantam Books.

Hanin, Y. (2007). Emotions in sport: current issues and perspectives. Handbook of sport psychology, 31-58.

Laborde, S., \& Dosseville, F. (2015). Emotional Intelligence in sport and exercise: A systematic review. Scandinavian Journal of Medicine and Science in Sports.

Laborde, S., Dosseville, F., Guillén, F., \& Chávez, E. (2014). Validity of the trait emotional intelligence questionnaire in sports and its links with performance satisfaction. Pshychology of Sports and Exercise, 15, 481-490.

Lane, A., Meyer, B., Devonport, T., Davies, K., Thelwell, R., Gill, G., ... Weston, N. (2009). Validity of the emotional intelligence scale for use in sport. Journal os Sports Science and Medicine, 8, 289-295.

Leal, E., Aparicio, D., Luti, Y., Acosta, L., Finol, F., Rojas, E., . . Velasco, M. (2009). Actividad física y enfermedad cardiovascular. Revista Latinoamericana de Hipertensión, 4(1), 2-16.

Lopes, P., Barsade, S., Nezlek, J., Elfenbein, H., Straus, R., \& Salovey, P. (2003). Ability to read emotions in group negotiations: Help or hindrance?.

Lopes, P., Côté, S., \& Salovey, P. (2014). Un modelo de habilidad de la inteligencia emocional: implicaciones para la evaluación y el entrenamiento. En J. Mestre Navas, \& P. Fernández-Berrocal, Manual de inteligencia emocional (págs. 217-234). Madrid: Editorial Pirámide.

Mayer, J., \& Geher, G. (1996). Emotional Intelligence and the identification of emotion. Intelligence(22), 89-113.

Mayer, J., \& Salovey, P. (1997). What is Emotional Intelligence? Emotional Development and Emotional Intelligence: Implications for Educators, 3-31.

Mayer, J., Salovey, P., \& Caruso, D. (2002). Mayer-Salovey-Caruso Emotional Intelligence Test (MSCEIT), Version 2.0. (T. M.H. Systems, Ed.)

Mestre, J., Comunian, A., \& Comunian , M. (2014). Inteligencia emocional: una revisión a sus primeros quince años y un acercamiento conceptual desde los procesos psicológicos. En J. M. Mestre Navas, \& P. Fernández-Berrocal, Manual de inteligencia emocional. (págs. 48-68). Madrid: Editorial Pirámide.

Pérez-González, J., Petrides, K., \& Furnham, A. (2014). La medida de la inteligencia emocional rasgo. En J. Mestre Navas, \& P. Fernández-Berrocal, Manual de inteligencia emocional (págs. 82-97). MAdrid: Ediciones Pirámide.

Petrides, K., Pérez, J., \& Furnham, A. (2003). The Trait Emotional Intelligence Questionnaire (TEIQue): A measure of emotional self-efficacy. Symposium conducted at the 11th Biennial Meeting of the International Society for the Study of the Individual Differences. Graz, Austria.

Ros Martínez, A., Moya-Faz, F., \& Garcés de Los Fayos Ruiz, E. (2013). Inteligencia emocional y deporte: situación actual del estado de la investigación. Cuadernos de Psicología del Deporte, 13(1), 105-112.

Saklofske, D., Austin, E., Rohr, B., \& Andrews , J. (2007). Personality, Emotional Intelligence and Exercise. Journal of Health Phychology, 937-948.

Salovey, P., \& Mayer, J. (1990). Emotional Intelligence. Imagination, Cognition \& Personality, 9, 185-211.

Schutte,, N., Malouff, J., Hall, L., Haggerty, D., Cooper, J., Golden, C., \& Dornheim, L. (2001). Development and validation of a mesure of emotional intelligence. Personality and Individual Differences(25), 167-177.

Shuk-Fong Li, G., Lu, F., \& Hsiu-Hua Wang, A. (2009). Exploring the relationship of physical activity. emotional intelligence and health in Taiwan College students. Journal of exerciese Science and Fitness, 7(1), 55-63.

Sullivan, A. (1999). The Emotional Intelligence Scale for Children. Dissertations Abstracts Internacional, 60(1A), 68.

Travis, R., \& Lac, D. (2006). Ability-versus skill-based assessment of emotional intelligence. Psicothema, 18, 59-66.

Vaquero-Solís, M., Amado, D., Sánchez-Oliva, D., Sánchez, P., \& Iglesias-Gallego, D. (2018). Inteligencia emocional en la adolescencia: motivación y actividad física. Revista Internacional de Medicina y Ciencias de La Actividad Física y Del Deporte, $X$.

Wong, C., \& Law, K. (2002). The effects of leader and follower emotional intelligence on performance and attitude: An exploratory study. Leadership Quarterly, 13(5), 505-522. 